\title{
Susanne Dröge: Revision der Fortsetzungskartei in der Kaufakzession beendet
}

Die nach der Abbestellaktion, die im Jahr 1997 durchgeführt wurde, erforderlichen Nachbearbeitungen sind nun beendet.

D. h. es wurden alle Fortsetzungskarten auf einen eventuellen Abschluss hin geprüft und die Karten abgelegt, wenn lange nichts mehr erschienen ist bzw. der Verlag auf Anfrage hin einen Abschluss des Werkes gemeldet hat.

Weiterhin wurden alle Verweisungen auf Kardex, die Bibliothek für Zeitgeschichte, Tausch und Geschenk überprüft und auf den neuesten Stand gebracht.

In der letzten Phase wurden bei einer nochmaligen Durchsicht damals versehentlich nicht markierte Karten bearbeitet. Zur Erinnerung: abbestellte Titel sind orange und laufende mit grün markiert. Nach dieser letzten Prüfung sind alle Karten auch wieder in einem Alphabet, d.h. es sind alle formalen Untergruppen aufgelöst worden.

Zum guten Schluss haben wir die Kartei verdichtet, so dass wir auf ein Element verzichten konnten. Dieses Element steht jetzt in der Graphischen Sammlung und beherbergt die überformatigen Postkarten.

Die übrigen zwei Elemente stehen jetzt auch nicht mehr an ihrem angestammten Platz als Theke mitten im Raum, sondern gleich links am Waschbecken. Dort stand vorher unser zentraler PC, der durch die Umstellung auf Windows NT und die damit einhergehende Aufstockung der Geräte überflüssig wurde.

Alle Mitarbeiter sind herzlich eingeladen, alles vor Ort zu besichtigen und eifrig Vorakzession an der nun wieder übersichtlichen Kartei zu machen.

Alle, die Vorakzession machen, müssen bedenken, dass die Fortsetzungskartei auch weiterhin bei allen mehrbändigen Werken und allen Schriftenreihen zu prüfen ist. Zu beachten ist ferner auch, dass über Fortsetzung eingehende Werke nur hier und danach erst wieder im SWB bzW. Stopac nachgewiesen sind, in der Akzessionskartei wird kein Zettel mehr eingelegt. 